\title{
Modeling and Analysis of the Self-powered Device for Wireless Heart Rate Measurement
}

\author{
Aripriharta ${ }^{1,2, a}$, Muladi ${ }^{1,2, b^{*}}$, Nandang Mufti1 ${ }^{1,3, c}$, Ilham Ari Elbaith Zaeni2,d, I Made \\ Wirawan ${ }^{2, \mathrm{e}}$, Gwo Jiun Horng ${ }^{4, \mathrm{f}}$ \\ ${ }^{1}$ Centre of Advanced Materials for Renewable Energy (Camry), Universitas Negeri Malang, \\ Indonesia \\ ${ }^{2}$ Department of Electrical Engineering, Faculty of Engineering, Universitas Negeri Malang, \\ Malang, Indonesia \\ ${ }^{3}$ Department of Physics Science, Faculty of Mathematics and Natural Science, Universitas \\ Negeri Malang, Malang, Indonesia \\ ${ }^{4}$ Department of Computer Science and Information Engineering, Southern Taiwan \\ University of Science and Technology, Tainan, Taiwan \\ a*aripriharta.ft@um.ac.id, bmuladi@um.ac.id,cnandang.mufti.fmipa@um.ac.id, \\ dilham_aez@yahoo.com, emade.wirawan.ft@um.ac.id, fgrojium@mail.stust.edu.tw
}

\begin{abstract}
A new circuit model of the self-powered device for heart rate measurement is presented in this paper. This device consists of piezoelectric energy harvester (PEH), power management circuit (PMC) with energy storage, microcontroller, Photoplethysmography (PPG) sensor, and Wi-Fi module. The PEH is placed under the insole to harvest the pressure energy from human foot-step to generate ac power. In our model, a PEH is represented by a sine voltage source, where its parameters were taken from experiments with 20 volunteers. The PMC is simplified by a switch with gain $\delta$ placed in series with the main circuit. The model of the main circuit is RC elements in parallel, where $\mathrm{C}$ is the capacitance of the storage device, and $\mathrm{R}$ is the equivalent parallel resistance of the microcontroller, PPG sensor, and Wi-Fi modules, respectively. The value of $\mathrm{R}$ depends on the power and current absorbed by those modules during sleep, deep sleep, sense, and transmit modes which collected from the datasheet. Finally, the proposed circuit model of the self-powered device was built and simulated in SPICE. The simulation results were compared with the laboratory experiment using commercial devices. Based on the results, the proposed model had small gaps compared to the real self-powered devices in terms of average current, voltage, power and efficiency.
\end{abstract}

Keywords: ambient human energy, piezoelectric energy harvester, RC circuit model, selfpowered device, wireless PPG sensor

\section{Introduction}

Self-powered devices are broadly used in structure $[1,2]$ and medical or health monitoring application [3]. The term self-powered means that the device is battery-less which powered itself by scavenging the ambient energy from the environment. The tool that is used for scavenging the ambient energy is called energy harvester. The main advantage of these devices is maintenance-free and pollution-free since they are replacing battery [4].

A piezoelectric is commonly used for energy harvesters (EH) in biomechanical application because it can give higher power density [1]. Piezoelectric converts vibration into electrical energy or in reverse. Human locomotion produces sustainable energy, for example, a walking human gives force to the shoe insole which high enough to supply the 
self-powered device. Base on the review in [5] mentions that the harvested energy from a $68 \mathrm{~kg}$ walking man using piezoelectric is about $20 \mathrm{~mW}$ to $80 \mathrm{~mW}$ depending on the structure of piezoelectric. Meanwhile, the average power generated by a $1 \mathrm{~cm}^{3}$ piezoelectric is about $100 \mu \mathrm{W}[1,6]$ deep sleep mode in the microcontroller which reduced the power consumption from $\mathrm{mA}$ to $\mu \mathrm{A}$. Moreover, the development of PMC such as LTC3588 brings the PEH to become more efficient [7].

Some researchers [4, 8-10] has been focused on developed PEH model for wearable devices which is embedded in the shoe. Most of them derived a circuit model and tested their model through simulation and/or experiment. Only a few authors derived the mathematical model for computation and optimization with high complexity [11, 12]. For the best of our knowledge, none of the previous works were formulated the PEH circuit as the simple RC charging-discharging circuit. Thus, in this paper, we proposed a simple RC equivalent circuit for performance analysis of the self-powered devices for heart rate measurement. Finally, we conducted an experiment to validate our model.

In a medical application, the self-powered devices use for physiological measurements including but not limited to heart rate. Since the traditional measurement, the heart rate is commonly measured on the wrist. As the rapid development of the PPG sensor, the heart rate could be measured from the neck, ear or fingertip [13]. Our contribution in this paper is extended to the heart rate measurement, where the heart rate data are collected from dorsalis pedis located on feet. This proposal is aimed to make a compact wearable device which integrated with a shoe. Besides, it reduces the number of microcontrollers and wireless module carried by the human body. Until this far, there is none of the selfpowered embedded shoes was considered the heart rate measurement from the feet artery.

The rest of this paper is organized as follow. The next section is related works which continued by Method. Then, the results and analysis are presented. Finally, the summary of this research is described in the last section.

\section{Related Works}

The self-powered devices which used piezoelectric energy harvester (PEH) are broadly used in many applications, such as bridge vibration monitoring [1], applied forced to floor in building interior [2], telemedical care [3], and wearable applications, e.g. integrated shoe $[4,8-10,14]$. In this section, we describe the related works more specific to PEH circuit modeling and optimization for wearable devices.

A PEH consists of the piezoelectric, rectifier, storage capacitor and power management circuit [4]. Piezoelectric is a device to convert the mechanical energy to electrical energy or vice versa. Fig. 1 shows the commonly used piezoelectric for PEH application. The circular type of commercial piezoelectric is depicted in Fig. 1, which mostly ceramics. Fig. 1.b. shows the rectangular shape piezoelectrics (so-called cantilever beam) is available in many types of materials. In [1], cantilever beam which made from Micro Fiber Composite (MFC) material to accommodate the bridge structure, where MFC was represented by an 
equivalent voltage source with series capacitance. They were not considered the power management circuit in a simulation, but they used LTC3588 to developed their prototype for evaluating the feasibility of MFC for powering a vibration monitoring device.

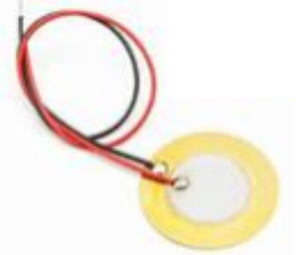

(a)

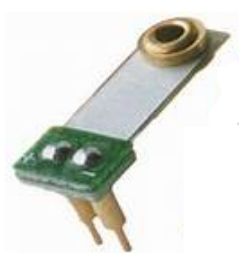

(b)

Fig. 1. Piezoelectric shapes, (a) circular shapes piezoelectric, (b) Rectangular shapes piezoelectric.

Oh, et.al [3] considered a complete mechanical-electrical equivalent circuit for replacing piezoelectric in the simulation. They developed a Complementary Metal-OxideSemiconductor (CMOS) controlled full-wave rectifier for averaging the AC voltage generated by piezoelectric. The rectifier was loaded by $45 \mathrm{k} \Omega$ and the generated output voltage is $703 \mathrm{mV}$.

The Heel Strike System that can generate power about $0.5 \mathrm{~W}$, was developed by researchers in [4]. PZT-5H ceramic [8] with a steel frame for embedded shoe and experience to generated $1.43 \mathrm{~mW}$ of electrical power. A nonlinear PEH under resonance and nonresonance conditions had been reported in [9]. The numerical simulations [10] and experiments of eight and six stacks of PEH embedded shoe was generated the average power outputs reached $20 \mathrm{~mW} / \mathrm{shoe}$ on a given walking speed about $5.6 \mathrm{~km} / \mathrm{h}$.

MFC material for replacing piezoelectric membrane due to high flexibility in large deformation have studied in [15], where two piezoelectric materials (PZT-5H and PZT-8) were placed into the sole to form a sandwich PEH structure. Moreover, computer software was used to observe the parameters of the sandwich-type of PEH and derived Lagrange approach to formulating a general electromechanical model of a sandwich PEH. The sandwich PEH could generate electrical power until $1.42 \mathrm{~mW}$ by using $0.4 \%$ of the applied force from $90 \mathrm{~kg}$ mass.
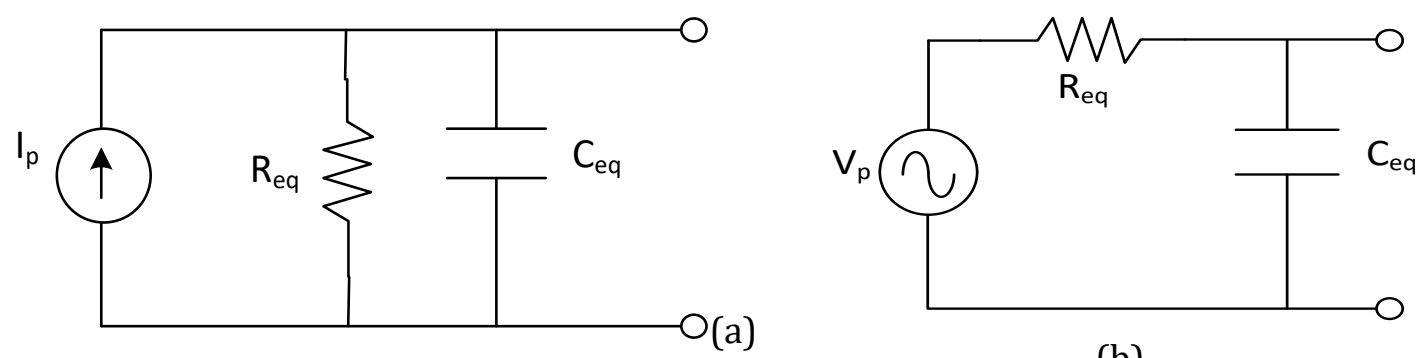

(b)

Fig. 2. PEH equivalent circuits as a current source (a) and as a voltage source (b).

PEH is commonly represented by a voltage or current source as depicted in Fig. 2. The equivalent circuits for representing piezoelectric in PEH are current source model and voltage source model, which are shown in Fig. 2.a. and Fig. 2.b., respectively. The works of 
$[1,2]$ in literature used voltage source model, and some of them used a current source $[3,5]$. The voltage and current sources in Fig. 2 are sinusoidal voltage, where the maximum RMS values of $I_{p}$ and $V_{p}$ occur at the resonant frequency of piezoelectric. Since the piezoelectric generated ac voltage; therefore, a bridge rectifier circuit used to convert the PEH output into a dc voltage. Moreover, some works have studied this model using an external resistor for tested PEH.

The works in the modeling of the power management circuit had been reported in the literature [11-12, 17-21]. Most of them used DC to DC converter to manage the generated voltage by the piezo generator. Moreover, the PEH circuit was optimized by Ottman, G. K., et. al. [11]. The structure of PEH and the axial deformation of PEH was introduced in the literature [20].

Comparison and contrast. The following are the comparisons and contrasts of our works with the previous state of the arts.

- Contrasting with the previous works in literature that exploited a cantilever beam which rectangular in shape [1-4, 9-12,15-22], we used the circular shape of piezoelectric.

- We used a voltage source equivalent circuit without considering $\mathrm{R}_{\text {eq }}$ and $\mathrm{C}_{\mathrm{eq}}$ as introduced by Whitaker [7].

- Our self-powered device operates under a deep sleep mode $[23,24]$ to decrease power consumptions. Meanwhile, the works in $[1,14,18]$ had reported that the sleep mode was applied to all nodes.

- We used SPICE for simulation. But, we derived the simulation parameter from datasheets and experiments with 20 volunteers.

- We validated the obtained results with NodeMCU [21] powered by the popular PEH chip (LTC3588) [1].

Novelties. The ultimate novelties of our works are as follow. The first is to simplify the model of self-powered devices, especially for load model which represented by resistors. We derived the resistances of each resistor for replacing microcontroller, sensor and transceiver modules. The second is the heart rate collection method. We collected the heart rate using the PPG sensor through dorsalis pedis artery located on feet. The third is a new model for a power management circuit which formed by a switch with gain $\delta$. The details of this model will explain in the next section.

\section{Method}

\subsection{System Description.}

Fig. 3 shows the self-powered devices for wireless heart rate measurement. This device consists of $\mathrm{PEH}$, power management and control circuits (PMC), storage device, microcontroller, PPG sensor, and Wi-Fi transceiver which placed together on the insole. $\mathrm{PEH}$ composes by a piezoelectric and bridge rectifier. The walking forces generate AC voltage to the piezoelectric terminals. A rectifier converts ac power generated by piezoelectric to DC power for charging a supercapacitor $3300 \mathrm{uF} / 10 \mathrm{~V}$. A PMC manages 
the power delivered to the load. The load consists of a microcontroller, PPG sensor, and Wi-Fi transceiver. The power ratings of those parts are listed in Table 1, where these data were collected from datasheet [1, 23-24].

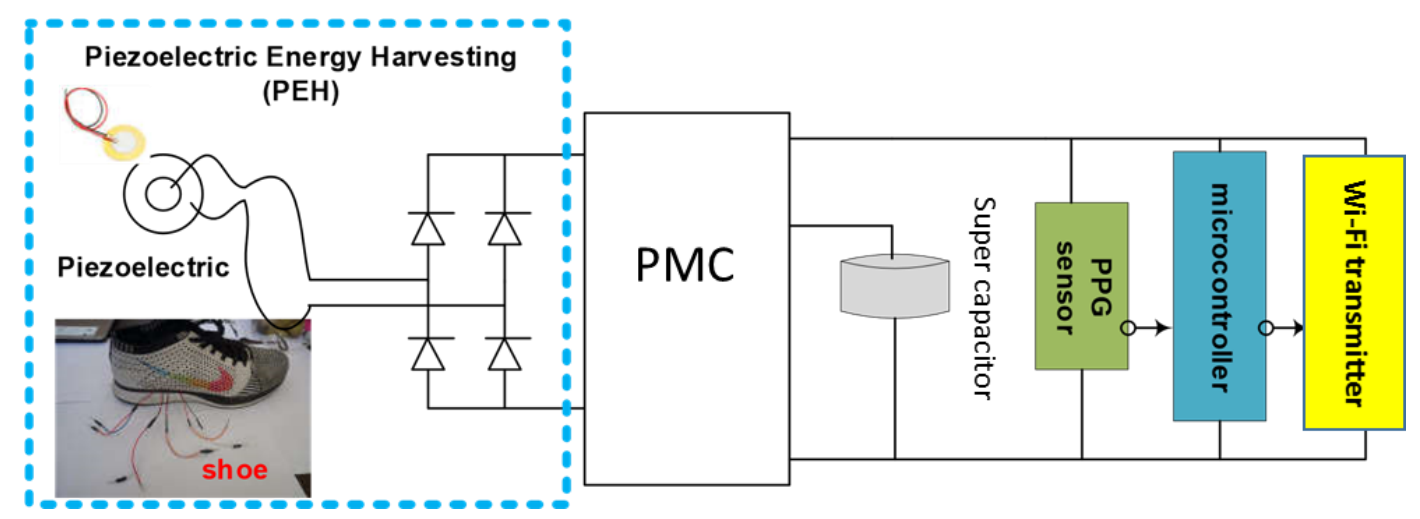

Fig. 3. A self-powered device for wireless heart rate measurement.

Table 1 Operating mode of the self-powered devices

\begin{tabular}{cccc}
\hline Task & Active Mode & $\begin{array}{c}\text { Time } \\
\text { duration }\end{array}$ & $\begin{array}{c}\text { Current } \\
\text { Consumption }\end{array}$ \\
\hline Sleep mode & microcontroller & $1000[\mathrm{~ms}]$ & $100[\mu \mathrm{A}]$ \\
\hline Wake up & $\begin{array}{c}\text { microcontroller, Wi-Fi } \\
\text { transmitter }\end{array}$ & $2[\mathrm{~ms}]$ & $7[\mathrm{~mA}]$ \\
\hline $\begin{array}{c}\text { Read heart rate data } \\
\text { using PPG sensor }\end{array}$ & microcontroller, PPG sensor & $3000[\mathrm{~ms}]$ & $20[\mathrm{~mA}]$ \\
\hline $\begin{array}{c}\text { Transmitting heart } \\
\text { rate }\end{array}$ & $\begin{array}{c}\text { microcontroller, Wi-Fi } \\
\text { transmitter }\end{array}$ & $30[\mathrm{~ms}]$ & $18[\mathrm{~mA}]$ \\
\hline Deep sleep & RTC & $3600[\mathrm{~ms}]$ & $15[\mu \mathrm{A}]$ \\
\hline
\end{tabular}

\subsection{Dorsalis pedis heart rate measurement.}

We proposed a new heart rate measurement through the dorsalis pedis artery which is located on feet (Fig. 4.a.). The PPG sensor is placed on the top of feet as shown in Fig. 4.a. The measured heart rate data send to the cloud data-storage through a microcontroller and Wi-Fi module. The user can monitor the heart rate from a smartphone as depicted in Fig. 4.b. 


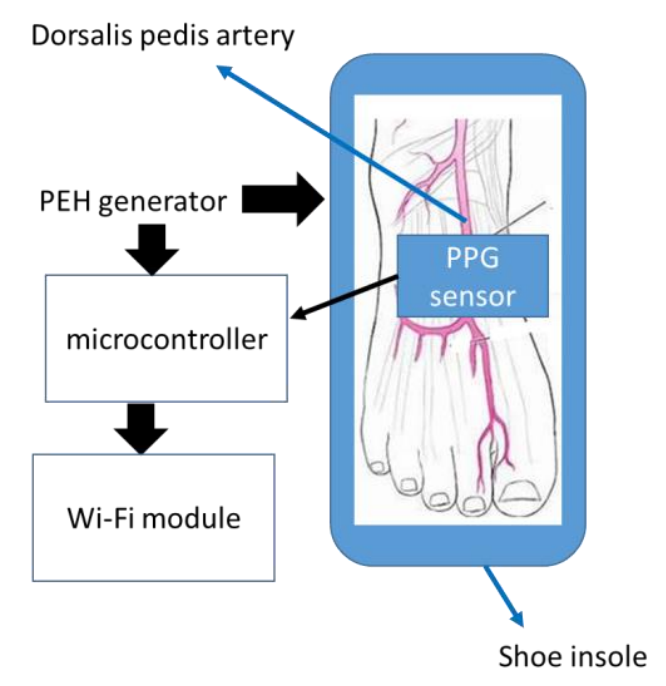

(a)

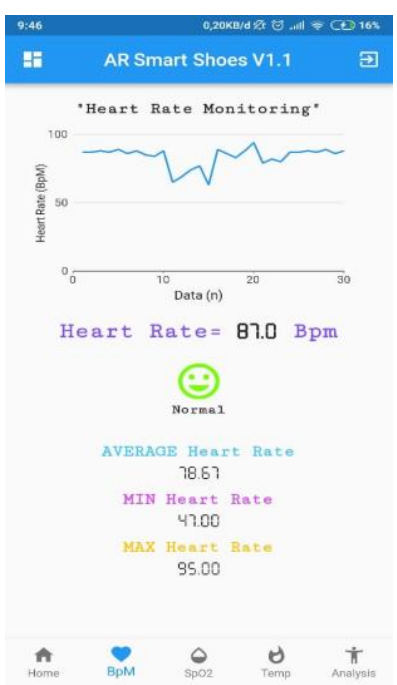

(b)

Fig. 4. Wireless heart rate measurement, (a) PPG sensor placement, (b) Heart rate measurement displayed on Smartphone.

\subsection{Modeling}

The following steps are the development process of our self-powered model. The first step is simplifying the PEH model, which a piezoelectric and bridge rectifier as PEH (Fig. 3 ), to a voltage source with $\mathrm{V}_{\mathrm{PEH}}=0.367 . \mathrm{V}_{\mathrm{PZ}}$. The value of 0.367 determined the ratio of rectification by the bridge diode. The second step is modeling the PMC. A single-pole, double-throw (SPDT) switch replaces PMC. This switch has gain denoted by $\delta$, where $\delta$ is a real number between 0 and 1 .

The last step is derived from the equivalent resistors for the microcontroller, PPG sensor, and Wi-Fi transmitter modules while running a specific task, where $\mathrm{R}_{\text {Sleep }}, \mathrm{R}_{\text {Wakeup, }}$ $R_{\text {Read, }} t_{\text {ransmit }}, R_{\text {Deepsleep }}$ respectively is their resistances. $S_{1}, S_{2}, S_{3}, S_{4}, S_{5}$ and $T_{1}, T_{2}, T_{3}, T_{4}, T_{5}$ are representing the switches and timers for activating each task, respectively. The length of the timers is equal to the time duration of tasks in Table 1. The proposed RC equivalent circuit for our self-powered devices is depicted in Fig. 5.

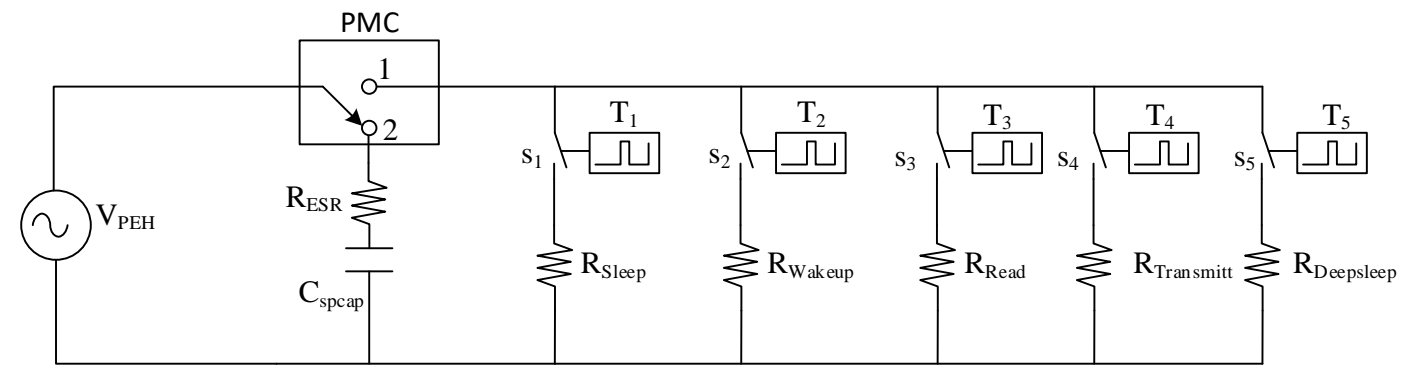

Fig. 5. RC equivalent model of our self-powered device.

\subsection{Simulation}

The proposed model of our self-powered device (Fig. 5) is simulated in the Simulator Program with Integrated Circuit Emphasis (SPICE) for $10 \mathrm{~s}$. The parameters used for the 
simulation are shown in Table 2. We measured the open voltage on the rectifier, $\mathrm{V}_{\mathrm{PZ}}$ which is given from the average of 20 samples. This approach gives more accuracy since the voltage dropped in rectifier are involved in measurement rather than calculated.

Table 2 Simulation Parameters

\begin{tabular}{|c|c|c|}
\hline Parameters & Values & Description \\
\hline $\mathrm{V}_{\mathrm{PZ}}$ & $11[\mathrm{~V}]$ & $\begin{array}{l}\text { The maximum output voltage measured at the piezoelectric } \\
\text { terminal }\end{array}$ \\
\hline $\mathrm{V}_{\mathrm{PEH}}$ & $0.637 . V_{\mathrm{PZ}}$ & $\begin{array}{l}\text { PEH output voltage measured from the output terminal of the } \\
\text { bridge rectifier (Fig. } 3 \text { ). } V_{\mathrm{PEH}}=(2 / \pi) . \mathrm{V}_{\mathrm{PZ}} \text {, where the rectification } \\
\text { ratio is } 2 / \pi \text { or equal to } 0.637 \text {. }\end{array}$ \\
\hline $\mathrm{R}_{\text {Sleep }}$ & $33 \mathrm{k} \Omega$ & The resistance of self-power device during sleep mode \\
\hline $\mathrm{R}_{\text {Wakeup }}$ & $471 \Omega$ & The resistance of self-power device during wake up mode \\
\hline $\mathrm{R}_{\text {Read }}$ & $33 \Omega$ & The resistance of self-power device during reading heart rate \\
\hline $\mathrm{R}_{\text {Transmit }}$ & $33 \Omega$ & The resistance of self-power device during data transmission \\
\hline $\mathrm{R}_{\text {Deepsleep }}$ & $330 \mathrm{k} \Omega$ & The resistance of self-power device during deep sleep \\
\hline$\delta$ & 0.9 & $\begin{array}{l}\text { PMC gain. This value was selected to guarantee the minimum } \\
\text { voltage required by the microcontroller and another module. }\end{array}$ \\
\hline
\end{tabular}

The volunteers are divided into 3 groups based on their weight. Volunteers in Group A had an average weight of $50 \mathrm{~kg}$ with a deviation of $3.2 \mathrm{~kg}$. Group B is volunteers with an average weight of $60 \mathrm{~kg}$ with a deviation of $5.6 \mathrm{~kg}$. The average volunteer's weight in Group C is $70 \mathrm{~kg}$ and the deviation is $2.5 \mathrm{~kg}$. The last stage is to analyze the influence of the difference in mass on the current and voltage produced by PEH. In addition, we also display PEH characteristics and calculate efficiency. The rest of the parameters used in our simulation are listed in Table 2 . The values of $R_{\text {Sleep }}, R_{\text {Wakeup }}, R_{\text {Read, }}, R_{\text {Transmit, }} R_{\text {Deepsleep }}$ were collected from direct measurement to the device.

\section{Results and Analysis}

The proposed self-powered device was developed and tested in several times using SPICE. Each simulation has done for $10 \mathrm{~s}$. The results of the simulations and performance analysis of the self-powered devices are presented in this section. Fig. 6 shows the averages of PEH output voltage, $\mathrm{V}_{\mathrm{PEH}}$ generated by the walking samples for each group. As the applied forces to the PEH is increased the output voltage is also increased; thus, Group $\mathrm{C}$ or the walking $70 \pm 2.5 \mathrm{~kg}$ mass gives the highest open voltage at the piezo terminal. The smallest average voltage measured on PEH output is given by the forces from $50 \pm 3.2 \mathrm{~kg}$ mass (Group A).

The short circuit current of PEH, Isc is depicted in Fig. 7. The highest short circuit current ( $\mathrm{I}_{\mathrm{SC}}$ ) is drawn by the highest mass which belongs to Group C, and the lowest $\mathrm{I}_{\mathrm{SC}}$ produced by the lowest pressure caused by the lowest mass (Group A). The characteristic 
of our PEH which is taken from the experiment is shown in Fig. 8. The short circuit current ( $\mathrm{Isc}_{\mathrm{sc}}$ ) of single circular piezoelectric is more than $10 \mu \mathrm{A}$ with the open-circuit voltage is $2 \mathrm{~V}$. When Isc decreases to $4.5 \mu \mathrm{A}$ and the voltage was $20 \mathrm{~V}$, the generated power was $90 \mu \mathrm{W}$. This condition achieved by hard force on shoe insole by $70 \pm 2.5 \mathrm{~kg}$ (Group C) walking mass. We add two more pieces of piezoelectric which connected in parallel to increase the power. Since the sufficient voltage for the charging capacitor is $11 \mathrm{~V}$; therefore, the given power is $280.5 \mu \mathrm{W}$, enough for wireless heart rate transmission. A supercapacitor will back up the power while our device read data from PPG sensor, wake up mode and transmitting data through Wi-Fi.

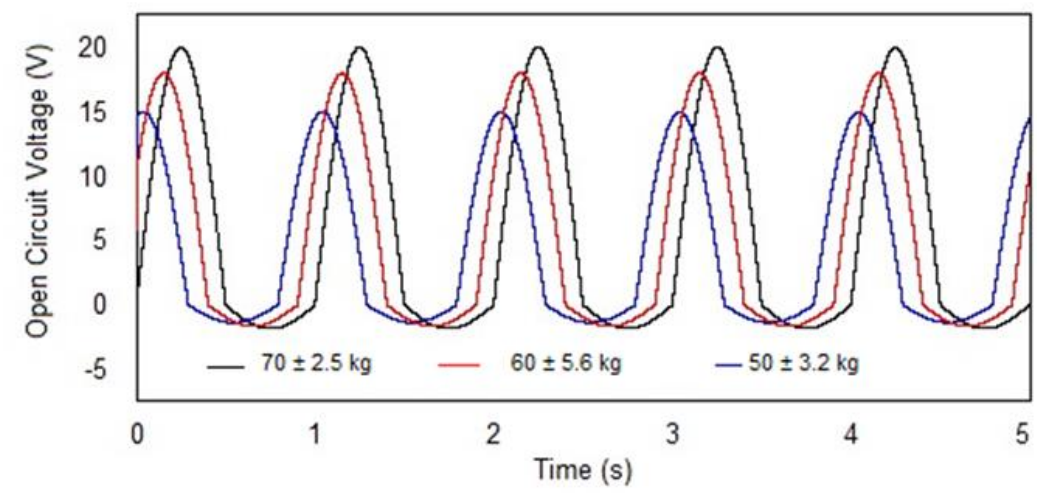

Fig. 6. PEH output voltage

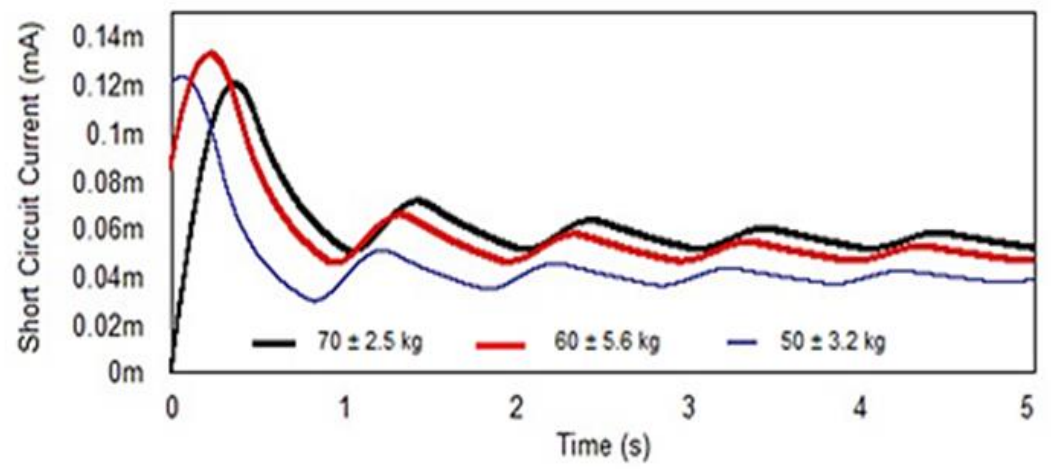

Fig. 7. Short circuit current of PEH.

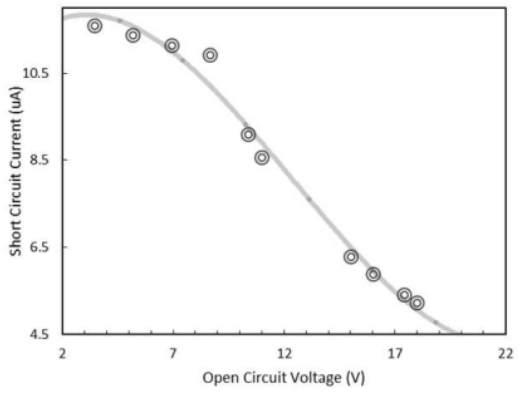

Fig. 8. PEH Characteristic.
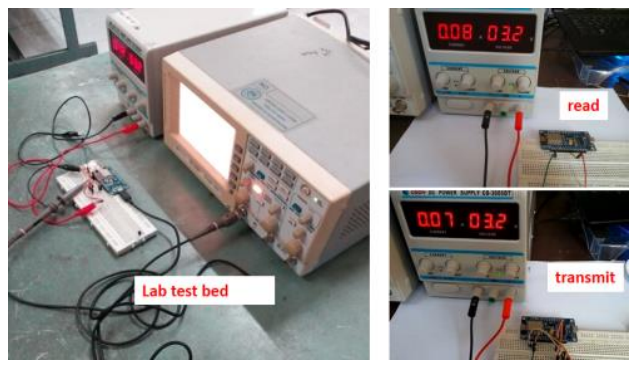

Fig. 9. A lab testbed with the standard device (NodeMCU). 
The test bed (Fig. 9) results of the current consumption of a standard device commercially available in the market, such as NodeMCU [23-24]. The minimum current and voltage required for operating NodeMCU are shown in Fig. 9. The average current required for running NodeMCU and our model are listed in Table 3. During sleep mode, the proposed model consumed $100 \mathrm{~mA}$ for reading the PPG sensor which is lower $10 \mathrm{~mA}$ than the current consumed by NodeMCU. The current needed for the operation of writing and data transmission task is equal to the datasheet of NodeMCU. These results show that our proposal can reduce power consumption under sleep mode. Moreover, the simulation results of our model for deep sleep mode are depicted in Fig. 10. The red line represents the current drawn by NodeMCU under deep sleep mode, while the blue line is our model. From this figure, we can conclude that our model is better $5 \mu \mathrm{A}$ than the NodeMCU.

Table 3 Current consumption comparison

\begin{tabular}{cccc}
\hline Item & Read (A) & Write $(\mathrm{A})$ & Transmit $(\mathrm{A})$ \\
\hline Node MCU test & 0.11 & 0.11 & 0.12 \\
\hline $\begin{array}{c}\text { NodeMCU } \\
\text { (datasheet) }\end{array}$ & 0.14 & 0.08 & 0.08 \\
\hline Our model & 0.1 & 0.08 & 0.08 \\
\hline
\end{tabular}

Table 4 Power consumption

\begin{tabular}{lccc}
\hline \multirow{2}{*}{ Mode } & \multicolumn{2}{c}{ Power [mW] } & Error \\
\cline { 2 - 3 } & Simulation & Testbed & $(\%)$ \\
\hline Normal Mode [14] & 100 & 110 & 10 \\
\hline Sleep mode only [1] & 40.3 & 50 & 24.06 \\
\hline Deep Sleep Mode [our model] & 40 & 42 & 5 \\
\hline
\end{tabular}

Table 4 shows the power consumed by the devices for running sensing and transmitting task in 3 modes of operation (normal, sleep, and deep sleep) in both simulation and experiment. In the normal mode, the power absorbs by the device under simulation test is $100 \mathrm{~mW}$. The experiment for the same operating mode gives $110 \mathrm{~mW}$, so the error is $10 \%$. The smallest error or the difference between simulation and testbed is achieved by our model is $5 \%$. 


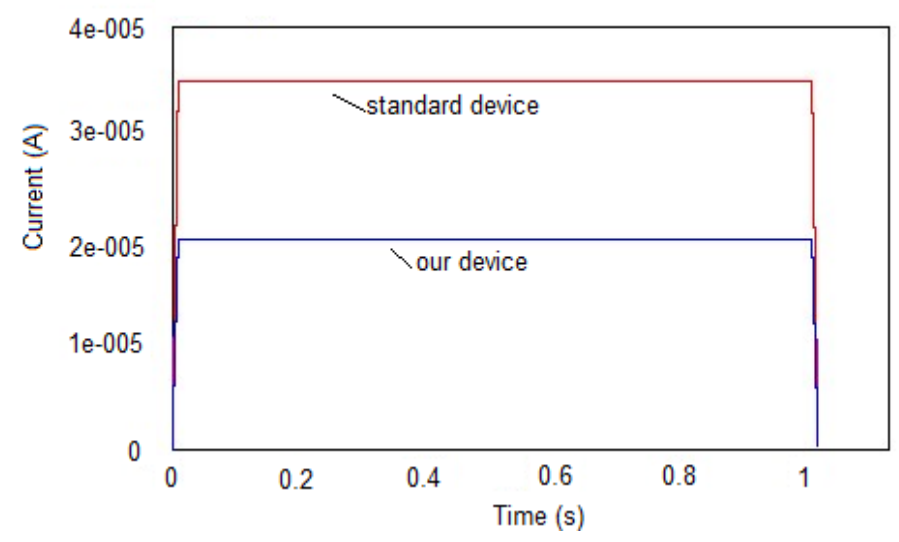

Fig. 10. Current consumption comparison chart.

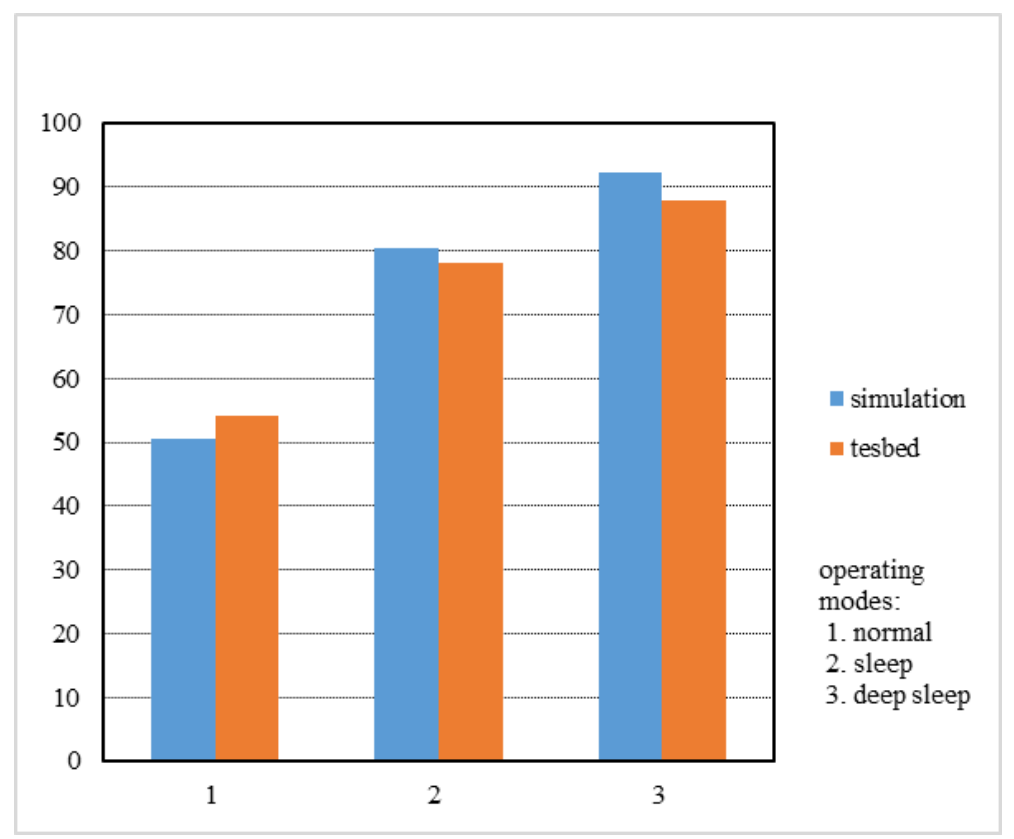

Fig. 11. Efficiency comparison of our model simulation) and standard device (testbed).

Fig. 11 shows the efficiency comparison of our model with a standard self-powered device (NodeMCU). The efficiency was calculated from the ratio of power absorbed by the device to the input power from $\mathrm{PEH}$, where those data were collected from several tests. In the test, the source voltage kept constant and both devices are performed in three modes, namely normal, sleep and deep sleep. Since the output power is proportional to the square of the current flow to the resistance; therefore, the smallest current dissipated the lowest power and gives the highest efficiency. Based on the above rationale, the highest efficiency is reached by our model under a deep sleep, because our device consumed lower current compared to the standard device, see Fig. 10.

The lowest efficiency given by our model is $50.2 \%$ which occurs in normal mode. In normal mode, our model absorbed more power for warming up, while the standard devices could run under very low power. Thus, our device has lower efficiency compare to 
NodeMCU. In sleep mode, the proposed self-power device gives higher efficiency equal to 80.5\%, which higher than the standard device. After warming up, our device could absorb lower current compared to NodeMCU, thus our model has lower power dissipation and higher efficiency. Our model gives higher efficiency for about $4.3 \%$ compared to the commercial device while it operates in deep sleep mode. As we can see from Fig. 10, the proposed devices have drawn lower current compared to the NodeMCU; therefore, our device could have higher efficiency.

\section{Conclusion}

The self-powered device for wireless heart rate measurement was modeled into a simple RC circuit. The simulation was built and ran under SPICE. The proposed model performs better than the commercial MCU with Wi-Fi module in both sleep and deep sleep modes. Furthermore, our RC equivalent model reduces the deep sleep current to 5 $\mu \mathrm{A}$. The proposed system could generate power more than $200 \mu \mathrm{W}$ which is surpassed the previous work in [1]. The proposed model is better to compare to the previous works and standard commercial device (NodeMCU).

This model can perform better than the standard model in term of current consumption. In the future, we will improve our proposal and derive the mathematical model by using an ordinary differential equation and solve by numerical computation. In addition, we will involve our Queen honey-bee migration (QHBM) [25] for optimization.

\section{References}

[1] W. Liu, Z. Wang, S. Qu, and R. Luo, Vibration energy harvesting and management for wireless sensor networks in bridge structural monitoring, IEEE sensors (2015) 1-4.

[2] A. M. Elhalwagy, M. Y. M. Ghoneem, and M. Elhadidi, Feasibility study for using piezoelectric energy harvesting floor in buildings' interior spaces, Energy Procedia 115 (2017) 114-126,

[3] T. Oh, S. K. Islam, G. To, and M. Mahfouz, A low-power cmos energy harvesting circuit for wearable sensors using piezoelectric transducers. J. Low Power Electron. Appl. 33 (2017) 1-11.

[4] C. A. Howells, Piezoelectric Energy Harvesting." Energy Conversion and Management 50 (2009) 1847-1850.

[5] R. Ahmed, F. Mir, and S. Banerjee. A review on energy harvesting approaches for renewable energies from ambient vibrations and acoustic waves using piezoelectricity. Smart Mater. Struct. 26 (2017) 1-27.

[6] Y. Han, Y. Cao, J. Zhao, Y. Yin, L. Ye, X. Wang, Z. You. A Self-Powered Insole for Human Motion Recognition. Sensors 16 (2016) 1502.

[7] M. Whitaker, Energy Harvester Produces Power from Local Environment, Eliminating Batteries in Wireless Sensors, LT Journal of Analog Innovation 20 (2010) 3-8 
[8] A. C. Turkmen, C. Celik, Energy harvesting with the piezoelectric material integrated shoe, Energy (2017) 1-45.

[9] Z. Yang, A. Erturk, J. Zu, On the effciency of piezoelectric energy harvesters, Extreme Mechanics Letters (2017) 1-24.

[10] Q. Feng, T.-B. Xu, and L. Zuo, Design, optimization, modeling and testing of a piezoelectric footwear energy harvester, Energy Conversion and Management 171 (2018) 1352-1364.

[11] G. K. Ottman, H. F. Hofmann, and G. A. Lesieutre, Optimized piezoelectric energy harvesting circuit using step-down converter in discontinuous conduction mode, IEEE transactions on power electronics 18 (2003) 698-703.

[12] L. Wu, X. Do, S. Lee, and D. S. Ha, A self-powered and optimal sshi circuit integrated with an active rectifier for piezoelectric energy harvesting, IEEE Transactions on Circuits and Systems I: Regular Papers 64 (2017) 537-549.

[13] G.J. Jong, Aripriharta, and GJ. Horng, The PPG physiological signal for heart rate variability analysis, Wireless Pers Commun 97 (2017): 5229-5276.

[14] P. Lynggaard, A self-supporting wireless IoT node that uses kinetic energy harvesting, In Internet of Things Business Models, Users, and Networks (2017) 1-6.

[15] X. Li, D. Upadrashta, K. Yu, and Y. Yang, Sandwich piezoelectric energy harvester: analytical modeling and experimental validation, Energy Conversion and Management 176 (2018) 69-85.

[16] J. Sankman and D. Ma, A 12-MW to 1.1-MW AIM piezoelectric energy harvester for time-varying vibrations with 450-na $\backslash \mathrm{bm}$ q, IEEE Transactions on Power Electronics 30 (2015) 632-643.

[17] A. Romani, R. P. Paganelli, E. Sangiorgi, and M. Tartagni, Joint modeling of piezoelectric transducers and power conversion circuits for energy harvesting applications, IEEE Sensors Journal 13 (2013) 916-925.

[18] K. Sushanta, and H. B. Nemade, Modeling and simulation of a piezoelectric vibration energy harvester, Procedia Engineering, International Conference on Vibration Problems 144 (2016) 568-575.

[19] T. R. Huq, and S. S. Williamson, Comprehensive comparative analysis of piezoelectric energy harvesting circuits for battery charging applications, In IECON 2013 - 39th Annual Conference of the IEEE Industrial Electronics Society (2013) 6698-6702.

[20] E. Alper, Assumed-modes modeling of piezoelectric energy harvesters: eulerbernoulli, rayleigh, and timoshenko models with axial deformations, Computers \& Structures 106-107 (2012) 214-227.

[21] A. M. Matos, J. M. Guedes, K. P. Jayachandran, and H. C. Rodrigues, Computational model for power optimization of piezoelectric vibration energy harvesters with material homogenization." Computers \& Structures 192 (2017) 144-156.

[22] J. Zhao and Y. Zheng, A Shoe-Embedded Piezoelectric Energy Harvester for Wearable Sensors, Sensors 14 (2014) 12497-12510. 
[23] Information on https://www.losant.com/blog/making-the-esp8266-low-poweredwith-deep-sleep.

[24] Information on https://www.electronicdesign.com/boards/take-simple-stepstoward-extreme-low-power-design.

[25] G.J. Jong, Aripriharta, Hendrick et al., A Novel Queen Honey Bee Migration (QHBM) Algorithm for Sink Repositioning in Wireless Sensor Network. Wireless Pers Commun 95 (2017) 3209.

\section{Authors Statement}

Aripriharta (idea, project leader, and funding support, draft paper), Muladi (co-funding, data analysis), Nandang Mufti (draft paper), Ilham Ari Elbaith Zaeni (method), I Made Wirawan (test manager, Gwo Jiun Horng (final manuscript)

\section{Acknowledgment}

This work is supported by PNBP UM 2019, Indonesia under contract of 20.3.71/UN32.14.1/LT/2019.

\section{Conflict of Interest}

The authors declare there is no compete interest.

\section{Ethic Clearance}

The data shown in this paper is clear through standard protocol of using human data for publication. 